\title{
Identification for Control: Simple Process Models
}

\author{
Lennart Ljung, \\ Division of Automatic Control \\ Department of Electrical Engineering \\ Linköpings universitet, SE-581 83 Linköping, Sweden \\ WWW: http://www. control.isy.liu.se \\ E-mail: Ljung@isy.liu.se, @isy.liu.se
}

3rd December 2003

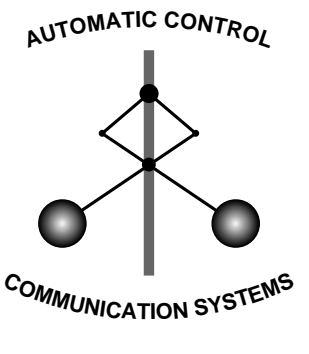

LINKÖPING

Report no.: LiTH-ISY-R-2465

Submitted to CDC'2002, Las Vegas

Technical reports from the Control \& Communication group in Linköping are available at http://www.control.isy.liu.se/publications. 


\title{
Identification for Control: Simple Process Models
}

\author{
Lennart Ljung \\ Div. of Automatic Control \\ Linköping University \\ SE-58183 Linköping, Sweden \\ email: ljung@isy.liu.se
}

\begin{abstract}
"Identification for control" in industrial practice most often means that a simple process model with two, three parameters are adjusted to a step response. These simple models are then used to tune PI- and possiby D-parameters in a basic controller. In this contribution we consider such simple process models from a system identification perspective. Some features of particular importance are the estimation focus and the input intersample behaviour, and we illustrate some of these aspects.
\end{abstract}

\section{Simple Process Models}

Simple process models of the kind Static Gain + Time Constant + Time Delay are dominating for control design in process industry. No doubt, the most common identification method in practice is to perform a step response experiment and adjust two or three parameters to the measured response. Nevertheless, such models have not been treated very much in the System Identification literature. The situation resembles that in control design, where the practice is dominated by PID-regulator tuning, which, with some exceptions, e.g. [12], is not widely treated in the literature.

The recent research area of Identification for control, e.g. [1], [13], [2], [14], [3] has produced many interesting results around the interplay between (reduced complexity) model estimation and control design. In industrial practice identification for control really is construction of a simple two- or three-parameter model, most often from a transient or possibly relay experiment, followed by tuning of a PI(D)-regulator, based on these twothree parameters.

In this contribution, we shall look into some aspects of how simple process models are identified, and how they approximate the true system.
2 Typical Models and Methods

Perhaps the most commonly used process model is

$$
G(s)=\frac{K}{1+s T_{p 1}} e^{-s T_{d}}
$$

Among variants of this model, we can have a model without delay $\left(T_{d}=0\right)$ :

$$
G(s)=\frac{K}{1+s T_{p 1}}
$$

and/or introduce an enforced integration (selfregulating process)

$$
G(s)=\frac{K}{s\left(1+s T_{p 1}\right)} e^{-s T_{d}}
$$

Moreover, on can postulate two real poles with or without a zero

$$
G(s)=\frac{K\left(1+s T_{z}\right)}{\left(1+s T_{p 1}\right)\left(1+s T_{p 2}\right)} e^{-s T_{d}}
$$

A further possibility is to allow resonant poles ("underdamped models"):

$$
G(s)=\frac{K\left(1+s T_{z}\right)}{1+2 \zeta s T_{r}+\left(s T_{r}\right)^{2}}
$$

Clearly a variety of models can be defined based on these components.

To the family of simple process models we could also count models that just define one point on the Nyquist curve

$$
G\left(i \omega^{*}\right)=A+B i
$$

where the frequency $\omega^{*}$ corresponds to "an interesting point", and may be part of what is identified. Alternatively, this model can be seen as a special case of (2), since given (6) one can uniquely determine (2).

Several papers and books discuss how to estimate models like (1) from transient response data (e.g. [12], [10], [15]). Most of the classical methods are graphical or semi-graphical, like finding the steepest tangent to the 
step response and calculate its intersection with the time axis, etc, or computing areas below the response curve and so on. See e.g. [12] for a recent overview of such approaches.

Relay experiments, [11], form another family of special identification experiments. By introducing a relay feedback, the system can be forced to self-oscillation, which will reveal where the Nyquist curve crosses the negative real axis. This gives a model of the type (6).

\section{A Prediction Error Identification Perspective}

In a standard system identification framework, e.g. [5], estimation of process models like (1) - (6) is of course no different from estimating any other parameterized linear model. Any of the models of the previous section can be written as

$$
G(s, \theta)
$$

where $\theta$ comprises the model parameters $K, T_{p 1}, T_{d}$ etc. To estimate the parameters we have collected a data set $Z^{N}=\{u(1), y(1), \ldots, u(N), y(N)\}$ of sampled inputs and outputs. Suppose the sampling interval is constant and equal to $T$. The model (7) is sampled with this sampling interval, according to the input intersample behavior (zero-order-hold, first-order-hold, bandlimited) giving the discrete time model

$$
G_{T}(q, \theta)
$$

( $q$ is the shift operator) and the model outputs

$$
\hat{y}(t \mid \theta)=G_{T}(q, \theta) u(t), \quad t=1, \ldots N .
$$

The parameters are then estimated by solving the nonlinear least squares problem

$$
\hat{\theta}_{N}=\arg \min _{\theta} \sum_{t=1}^{N}(y(t)-\hat{y}(t \mid \theta))^{2}
$$

It is also straightforward to include a model of additive noise

$$
y(t)=G(p, \theta) u(t)+H(p, \theta) e(t)
$$

where $p$ denotes the differentiation operator (replacing $s)$. Determining the proper sampled predictor from (11) and letting $\hat{y}(t \mid \theta)$ denote the corresponding predicted outputs, gives a method (10) that also estimates the noise model.

Moreover, an estimation focus can be defined as discussed in [4]. For a model with $H=1$, the estimation focus filter $L$ simply means that the inputs and outputs are first filtered through $L$.
The asymptotic properties of the estimated model are well known: Suppose the true frequency function for the sampled system is $G_{0}^{(T)}\left(e^{i \omega}\right)$ (or, more generally, the frequency function of the linear time invariant second order equivalent of the true system, see $[6])$. Then for $H=1$ we have $([5])$

$$
\begin{aligned}
\hat{\theta}_{N} \rightarrow \arg \min _{\theta} & \int_{-\pi}^{\pi}\left|G_{T}\left(e^{i \omega}, \theta\right)-G_{0}^{(T)}\left(e^{i \omega}\right)\right|^{2} \\
& \times \Phi_{u}(\omega)\left|L\left(e^{i \omega}\right)\right|^{2} d \omega
\end{aligned}
$$

Here $L$ is the "focus filter", and $\Phi_{u}$ is the input spectrum. The expression describes exactly in what way the simple process model like (1) approximates the true system. We also see how the focus filter may steer the fit to important frequency ranges.

In a sense, (12) also explains the success of simple process models. Even a three-parameter model like (1) has substantial "local flexibility". The delay term may pick up the true system's phase, even if there is no dead-time in the system. For successful control design it is often sufficient to have a rough picture of the Nyquist curve in a limited, but important frequency region, and (12) illustrates how this can be achieved.

The expression (12) also explains the type of model fit we get during a relay experiment with self-oscillation. The input spectrum will in such a case be narrowly concentrated to the resonance frequency, which means that any model, e.g. the simple first order model (2) will be closely fitted to the true frequency response at this frequency (cf (6)).

\section{Algorithmic Aspects}

Fitting simple process models to data, as described here, is just a special case of prediction error identification, and it can be easily implemented using, e.g. the idgrey model object in the SYSTEM IDENTIFICATION ToOlBox, [7]. There are, however a number of aspects that should be attended to for a successful implementation. Some of these will be discussed here, with some numerical illustrations to follow in the next section.

\subsection{Initial parameter values}

The non-linear least squares minimization problem (10) requires a starting point for the iterative minimization. Experience shows that the function to be minimized in (10) may have several local minima, so it is important to spend some effort in finding a good initial estimate.

The simplest way to obtain a good starting point is to run a black-box estimation step for a discrete time model, after the dead-time (with a delay of an integer number of sampling intervals) has been estimated 
separately. This black-box model could be estimated by a state-space subspace method or a prediction error method (n4sid or oe). Then this model is converted to continuous time. The conversion requires some attention:

- The process models (2)-(5) may have restrictions, like no zeros allowed, real poles etc., and a straightforward conversion might not be possible.

- The intersample input behavior must be taken into consideration at the conversion (see next subsection). Note that the intersample behavior also affects to orders that should be used in the black-box model. For example foh (first order hold) sampling gives rise to an extra direct term from input to output.

- To enforce an integration (to initialize models like (3)) experience shows that it is better to use the integrated (cumsum) input rather than the differentiated output when building the initial black box model. Note that this also affects the intersample behavior of the new input.

\subsection{Inter-sample input behavior}

The intersample input behavior could be given as piecewise constant (zoh = zero-order-hold) or piece-wise linear (foh = first-order-hold) or band-limited (bl) meaning that there are no frequencies in the continuous time input above the Nyquist frequency. Both when converting the initial black box model to continuous time, and when constructing the predicted output (9), the intersample behavior is important. For slowly sampled systems the bad effects of a wrong conversion may be substantial, as demonstrated in Section 5.2.

As a side remark, we may mention that the expressions for converting from discrete to continuous time under the foh assumption are, for some reason, seldom displayed in textbooks. Also, the function d2c in MATLAB's Control Systems Toolbox does not accept the foh option (in contrast to c2d.)

Anyway, here is how it could be done: First order hold sampling converts the continuous time model

$$
\begin{aligned}
& \dot{x}=A x+B u \\
& y=C x+D u
\end{aligned}
$$

to the discrete time model

$$
\begin{aligned}
x(t+T) & =F x(t)+G u(t) \\
y(t) & =C x(t)+H u(t) \\
F & =e^{A T} \\
G & =\Psi B \\
\Psi & =\frac{1}{T} \int_{0}^{T} e^{A \tau} \tau d \tau \\
H & =C+C \int_{0}^{T} e^{A \tau} d \tau-C G
\end{aligned}
$$

This means that given $F, G, H$ in (14ab) we find $A$ from (14c) in the usual way, then compute $\Psi$ from $(14 \mathrm{e})$ (which is the $G$-matrix for foh sampling with $B=I$ ) and then determine $B$ from (14d), etc.

\subsection{Delays that are not fractions of the sam- pling interval}

In models that allow a time-delay $T_{d}$, like (1) it is important not to restrict $T_{d}$ to be an integer number of sampling intervals. Otherwise the important flexibility to independently adjust the phase-shift to the true system is lost.

Estimating an arbitrary time delay just follows the general setup of Section 3. The formulas for sampling (7) to (8) when $T_{d}$ contains fractions of a sampling interval are well known, both for zoh and foh sampling. The only problem that needs to be handled when statespace representations are used, is to be observant on varying sizes of the state vector during the numerical minimization. See, e.g. [8] for a further discussion.

\subsection{Frequency domain data}

If the input-output data are given in the frequency domain as Fourier transforms, the prediction error approach to estimating process models still can be applied. See, e.g. Section 7.7 in [5] or chapters 5-8 in [9].

In fact, frequency domain data offer useful potentials also for other problems:

- The focus filter can be implemented as specific frequencies for which the fit should be made. These may not necessarily be known a priori, but could be selected from a preliminary model, like using frequencies that correspond to the Nyquist curve being in the third quadrant, or being close to the critical point -1 . Example:

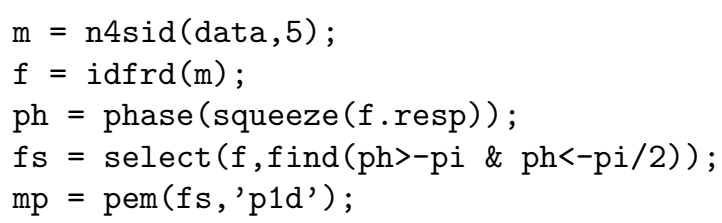


- If the intersample input behavior is band-limited, moving to the frequency domain will be the easiest way to handle the sampling. The FFT (discrete Fourier transform) of the input will then be equal to the Fourier transform of the underlying continuous time input signal. The FFT of the output will similarly correctly describe the continuous time Fourier transform of that part of the output that originates from the input, and we can directly fit a continuous time model:

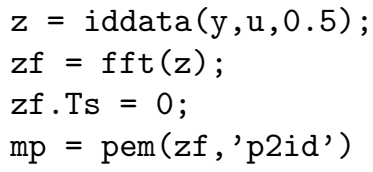

\section{Examples}

\subsection{An 8th order system}

In [12] the following model is considered:

$$
G(s)=\frac{1}{(1+s)^{8}}
$$

Step response data were simulated for this system with sampling interval 0.3 seconds. The process model (1) was fitted to these data using (10), giving the model

$$
G(s)=\frac{1.0053}{1+3.1691 s} e^{-5.2129 s}
$$

The step response and the Nyquist plot of this model are compared with the true system in Figure 1.

Despite the substantial difference in model complexity, the simple process model gives a good approximation. One might wish to have a better agreement around the phase crossover frequency, and for that reason we choose a focus filter

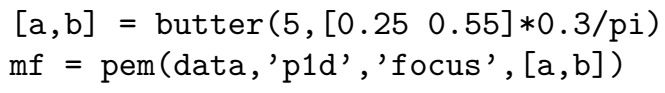

The resulting model is

$$
G(s)=\frac{1.0399}{1+3.9609 s} e^{-5.0202 s}
$$

and its time and frequency responses are also shown in Figure 1. The fit around the phase cross-over between the model (17) and the true system is now very close.

\subsection{Importance of input intersample behavior}

A 5th order linear system was randomly generated (using rss) and simulated with a white noise input, using first-order hold sampling, with a sampling interval of 1 sec. A portion of the data is shown in Figure 2. A
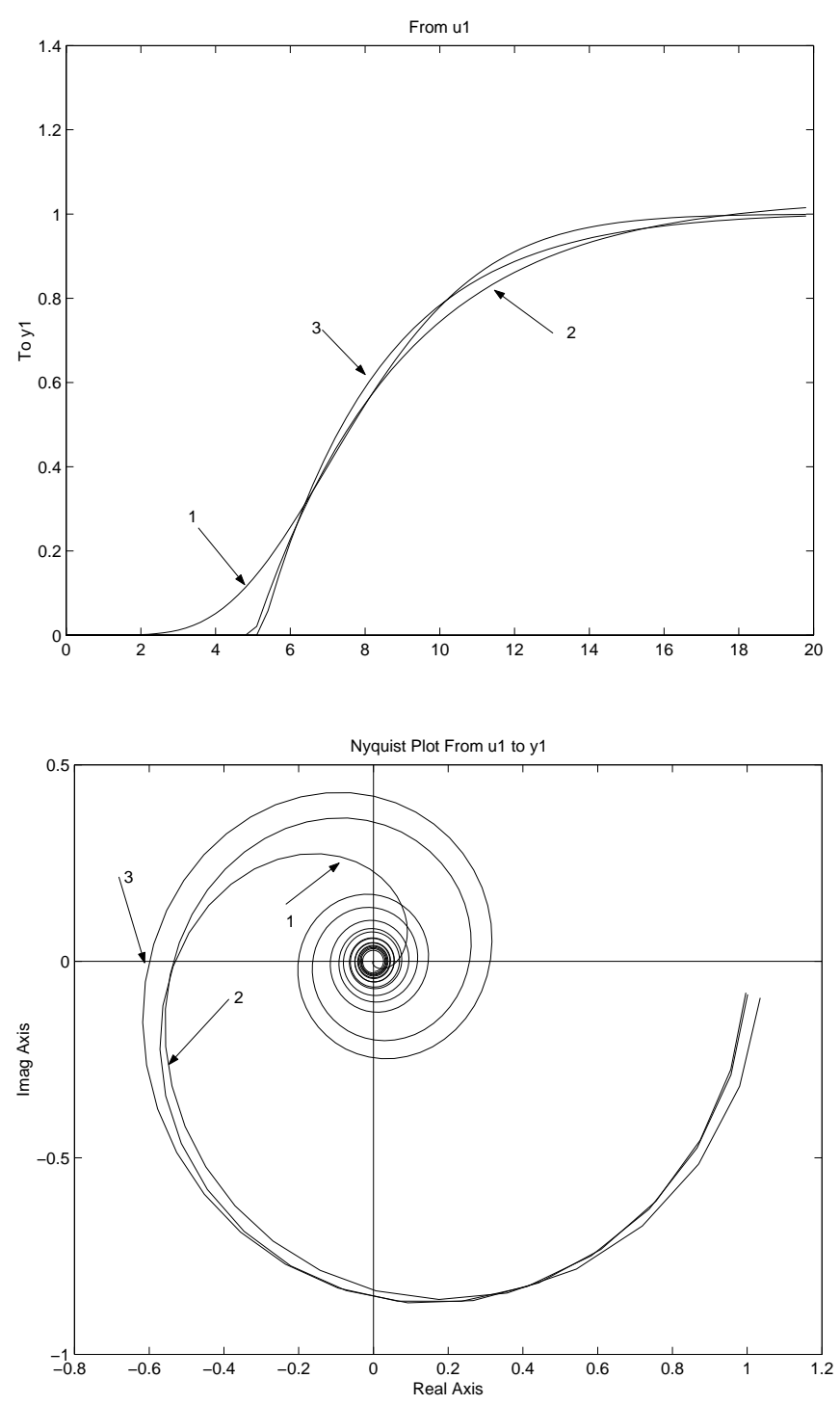

Figure 1: Step responses and Nyquist curves for the true system (curve 1), for the simple process model (17) (curve 2) and the model (16) (curve 3). 

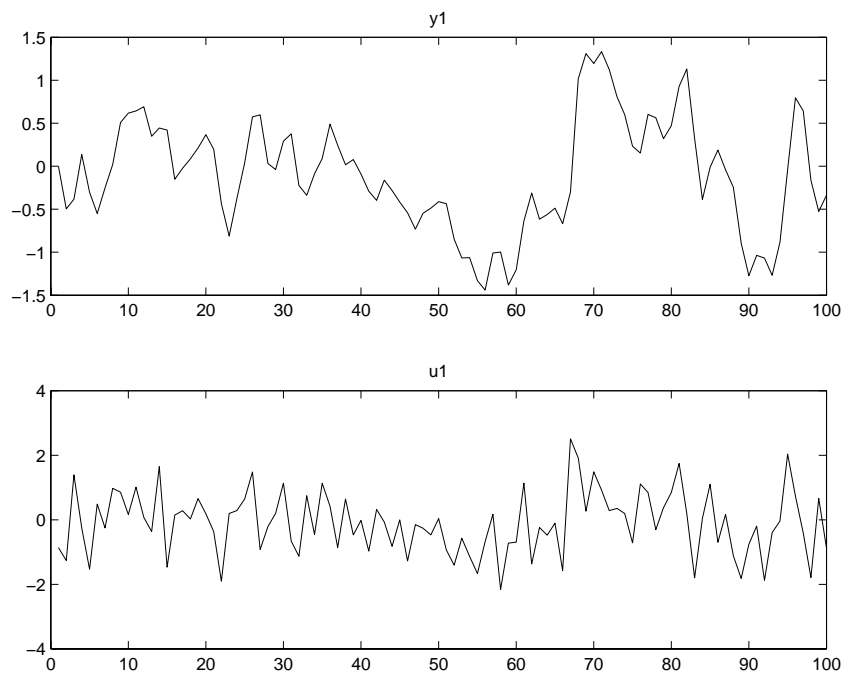

Figure 2: Portion of the data in Section 5.2.

simple model of the kind (1) was fitted to the data, correctly informing the estimation process that the input was foh. That gave the model

$$
G(s)=\frac{1.2633}{1+1.7519 s} e^{-0.15057 s}
$$

A model of the same structure was also estimated, now under the assumption that the intersample behavior was zero-order-hold. That gave the result

$$
G(s)=\frac{1.0898}{1+1.8246 s} e^{-0.0067 s}
$$

The step responses and the Nyquist plots of the two models are compared with the true curves in Figure 3. It is interesting to note that using the right intersample information gives a much better fit, despite the fact that the model set is not capable of describing the true system.

\section{Conclusions}

Building simple models for control design has typically been handled by ad hoc methods, and many of these have been quite successful. Approaching this estimation problem from a formal system identification viewpoint gives a number of advantages, though:

- The identification framework gives a packaged deal for analysis. Important insights like (12) about the character of the approximations involved follow from standard results.

- Also how to choose design variables, like the focus of the fit can be guided by such results.
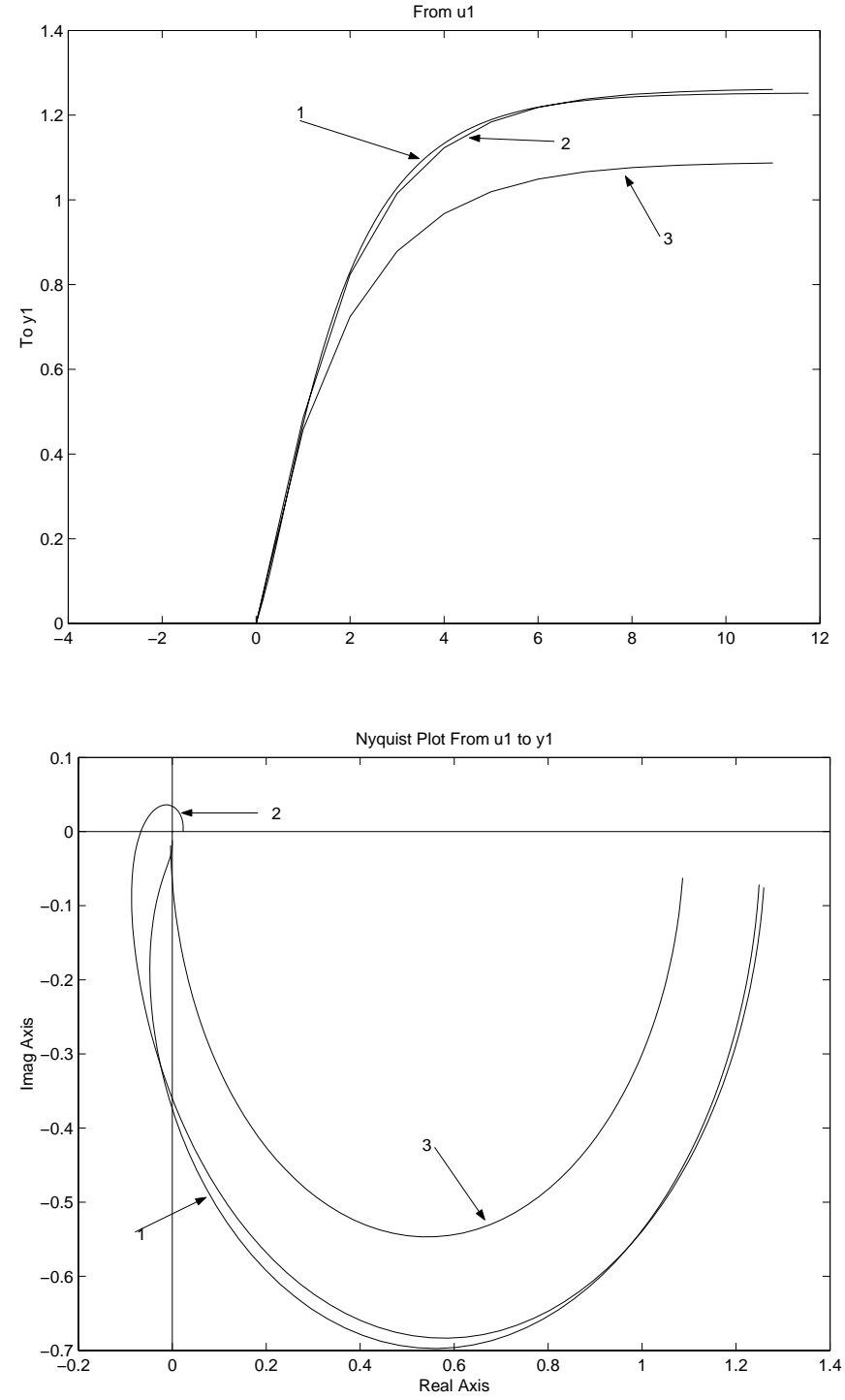

Figure 3: Step responses and Nyquist curves for the true system (curve 1), for the simple process model assuming FOH (curve 2) and the model assuming $\mathrm{ZOH}$ (curve 3). 
- For sampled data, a correct handling of the input intersample behaviour may be important. Again the formal approch has an concrete answer how to utilize that information. For bandlimited data, the possibility to use frequency domain representations may be essential.

The price for these features is that the minimization problem (10) may be non-trivial. This is especially pronounced when a time delay $T_{d}$ is included among the parameters. Due respect must thus be paid to the starting value and to numerical conditioning.

\section{References}

[1] M. Gevers. Towards a joint design of identification and control? In H L Trentelman and J C Willems, editors, Essays on control: Perspectives in the theory and its applications, ECC '93 Groningen, 1993.

[2] R.L. Kosut, G. C. Goodwin, and M. P. Polis (Eds). Special Issue on System Identification for Robust Control Design, IEEE Trans. Automatic Control, Vol 3\%. 1992.

[3] L. Ljung. Identification for control - what is there to learn? In Y. Yamamoto and S. Hara, editors, Learning, Control and Hybrid Systems, volume 241 of Springer Lecture Notes in Control and Information Sciences, pages 207-221, Berlin, Dec 1998. Springer Verlag.

[4] L. Ljung. Estimation focus in system identification: Prefiltering, noise models, and prediction. In IEEE Conference on Decision and Control, Dec 1999.

[5] L. Ljung. System Identification - Theory for the User. Prentice-Hall, Upper Saddle River, N.J., 2nd edition, 1999.

[6] L. Ljung. Estimating linear time invariant models of non-linear time-varying systems. European Journal of Control, 7(2-3):203-219, Sept 2001. Semi-plenary presentation at the European Control Conference, Sept 2001.

[7] L. Ljung. System Identification Toolbox for use with MatlaB. Version 6. The MathWorks, Inc, Natick, MA, 6th edition, 2003.

[8] P. Nagy and L. Ljung. Estimating time-delays via state-space identification methods. In Preprints 9th IFAC Symposium on System Identification and System Parameter Estimation, pages 1141-1144, Budapest, Hungary, July 1991.

[9] R. Pintelon and J. Schoukens. System Identification - A Frequency Domain Approach. IEEE Press, New York, 2001.

[10] H. Rake. Step response and frequency response methods. Automatica, 16:519-526, 1980.
[11] K. J. Åström and T. Hägglund. Automatic tuning of simple regulators with specifications on phase and amplitude margins. Automatica, 20:645-651, 1984.

[12] K. J. Åström and T. Hägglund. PID Controllers: Theory, Design, and Tuning. Instrument Society of America, Triangle Research Park, N.C., 2nd edition, 1995.

[13] P. M. J. van den Hof and R.J.P. Schrama. Identification and control-closed loop issues. Automatica, 31(12):1751-1770, December 1995.

[14] Z. Zang, R. R. Bitmead, and M. Gevers. Iterative Weighted Least-squares Identification and Weighted LQG Control Design. Automatica, 31(11):1577-1594, 1995.

[15] J. G. Ziegler, N. B. Nichols, and N.Y. Rochester. Process lags in automatic-control circuits. Trans. ASME, 65:443-444, July 1943. 
Abstract

Keywords: 


Avdelning, Institution
Division, Department
Division of Automatic Control
Department of Electrical Engineering

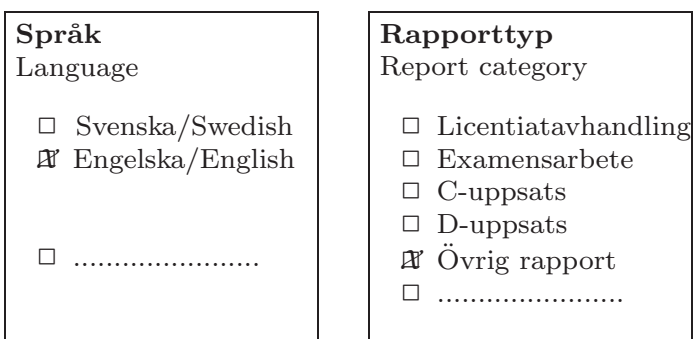

URL för elektronisk version

http://www. control.isy.liu.se

\begin{tabular}{|c|c|}
\hline ISBN & \\
\hline ISRN & \\
\hline $\begin{array}{l}\text { Serietitel och serienummer } \\
\text { Title of series, numbering }\end{array}$ & ISSN \\
\hline LiTH-ISY-R-2465 & \\
\hline
\end{tabular}

Titel

Title Identification for Control: Simple Process Models

Författare

Author Lennart Ljung, ,

Sammanfattning

Abstract

Nyckelord

Keywords 